\title{
Your Failure is My Opportunity - Eff ects of Elimination in Contests
}

Citation for published version (APA):

Mendel, M., Pieroth, F., \& Seel, C. (2019). Your Failure is My Opportunity - Eff ects of Elimination in Contests. Maastricht University, Graduate School of Business and Economics. GSBE Research Memoranda No. 016 https://doi.org/10.26481/umagsb.2019016

Document status and date:

Published: 06/06/2019

DOI:

10.26481/umagsb.2019016

Document Version:

Publisher's PDF, also known as Version of record

\section{Please check the document version of this publication:}

- A submitted manuscript is the version of the article upon submission and before peer-review. There can be important differences between the submitted version and the official published version of record.

People interested in the research are advised to contact the author for the final version of the publication, or visit the DOI to the publisher's website.

- The final author version and the galley proof are versions of the publication after peer review.

- The final published version features the final layout of the paper including the volume, issue and page numbers.

Link to publication

\footnotetext{
General rights rights.

- You may freely distribute the URL identifying the publication in the public portal. please follow below link for the End User Agreement:

www.umlib.nl/taverne-license

Take down policy

If you believe that this document breaches copyright please contact us at:

repository@maastrichtuniversity.nl

providing details and we will investigate your claim.
}

Copyright and moral rights for the publications made accessible in the public portal are retained by the authors and/or other copyright owners and it is a condition of accessing publications that users recognise and abide by the legal requirements associated with these

- Users may download and print one copy of any publication from the public portal for the purpose of private study or research.

- You may not further distribute the material or use it for any profit-making activity or commercial gain

If the publication is distributed under the terms of Article $25 \mathrm{fa}$ of the Dutch Copyright Act, indicated by the "Taverne" license above, 


\section{Maastricht University}

Moritz Mendel, Ferdinand Pieroth, Christian Seel

Your Failure is My Opportunity - Effects of Elimination in Contests

RM/19/016

\section{GSBE}

Maastricht University School of Business and Economics

Graduate School of Business and Economics

P.O Box 616

NL-6200 MD Maastricht

The Netherlands 


\title{
Your Failure is My Opportunity - Effects of Elimination in Contests*
}

\author{
Moritz Mendel ${ }^{\dagger} \quad$ Ferdinand Pieroth ${ }^{\ddagger} \quad$ Christian Seel ${ }^{\S}$
}

May 20, 2019

\begin{abstract}
In this paper, we consider a sequence of multi-prize all-pay auctions, where the contestants who exert the lowest efforts in a round are eliminated. We analyze if and how the behavior of contestants is influenced by the possibility that strong rivals are eliminated along the way. The conditions under which behavior is not influenced are rather restrictive. For cases where these conditions are not met, we derive equilibria in a two-period model. We compare our equilibrium predictions to those of a static model and a two-stage contest where the sequential structure is not announced in the first round.
\end{abstract}

KEYWORDS: Elimination contest; identity-dependent externality JEL classification: C72; D44

*We would like to thank José Alcalde, Carmen Beviá, Ronald Peeters, Arkadi Predtetchinski, Benny Moldovanu, participants of the SAET conference 2017 in Faro and seminar audiences in Alicante and Maastricht for useful comments. This paper supersedes an earlier draft called "Multi-Stage Intragroup Elimination Contests".

${ }^{\dagger}$ University of Bonn, Germany, E-Mail: moritz.mendel@uni-bonn.de

${ }^{\ddagger}$ Maastricht University, E-Mail: f.pieroth@maastrichtuniversity.nl

$\S$ Maastricht University, Department of Economics, E-Mail: c.seel@maastrichtuniversity.nl 


\section{Introduction}

Many dynamic contests feature multi-stage intragroup elimination procedures. Examples of such contests in sports include Formula 1 qualifying, where only the quickest drivers qualify for later qualification rounds, elimination races in track cycling where the slowest riders are eliminated in each round or athletics, where only the fastest runners qualify for the trials and the fastest runners in the trials qualify for World Championships or Olympic Games.

The scope of such contests reaches beyond applications in sports. For instance, consider scholarship applications where, in a first round, applicants submit a letter of motivation. Based on these letters, a subset of applicants is selected for the second round. The second round might be an assessment seminar, where the applicants can meet their peers and establish industry contacts. After the assessment seminar, the best applicants receive a scholarship. ${ }^{1}$ Other applications with similar features include runoff elections and different TV formats with popular voting (e.g., American Idol, Big Brother, Let's Dance, The Bachelor).

In these applications, all remaining contestants compete with each other in each round. The best-performing contestants qualify for the next round, while the remaining contestants are eliminated from the game. Additionally, some of the qualifying contestants may also receive an intermediate prize in the given round. For example, think of an additional prize awarded to the winner of a round in elimination races.

Such contest have for instance been studied in a Tullock lottery framework by Fu and Lu (2012) and Arve and Chiappinelli (2018) and for contests with noise by Altmann, Falk, and Wibral (2012) and Delfgaauw, Dur, Non, and Verbeke (2015). ${ }^{2}$ Our analysis differs in two aspects from these papers. First, in each stage an all-pay auction with complete information is played.

\footnotetext{
${ }^{1}$ Such a scheme is used by the German National Academic Foundation.

${ }^{2}$ The framework differs from elimination contests in which the set of contestants is split into subcontests in each round; see, e.g., Rosen (1986), Moldovanu and Sela (2006), and Groh, Moldovanu, Sela, and Sunde (2012). In those models, the winners of the subcontests compete against each other in later rounds. As an application, think of a tennis tournaments where pairs of players compete in a knock-out format in each round.
} 
Much more importantly, all of the mentioned papers avoid the analysis of a major effect: the expected gain from qualification for later rounds might depend on the identity of the other qualifiers. For instance, in athletics, it is easier to win a gold medal if a top contender did not qualify for the final. This problem disappears in the previous literature as contestants are either assumed to be symmetric or identity-dependent differences do not carry over to future rounds. In this paper, we explore if and how this additional factor influences equilibrium behavior.

The equilibrium analysis is ordered in decreasing level of generality. Proposition 2 derives conditions such that behavior is unaffected by the incentives to qualify, i.e., in the unique subgame-perfect equilibrium, the Nash equilibrium of the static multi-prize all-pay auction found in Clark and Riis (1998) is played among the qualified players in every round. These conditions are (i) there are fewer qualification spots than prizes in each round (except for the last), (ii) the order of valuations is constant across players and rounds and (iii) either the sequence of prizes is decreasing or a specific tie-breaking is used.

If at least one of the three conditions fails, behavior might be affected by the extra incentive to qualify. In particular, if the first condition fails behavior is always affected by expected future gains. We split these gains up into two parts. First, there is a qualification payoff, i.e., the future expected payoff when the strongest contestants qualify. The second effect is more subtle. The externality payoff reflects the well-known hope of a sportsman that strong rivals are eliminated along the way in order to have an easier final.

In contrast to the literature, the externality payoff depends on the own effort in the current period, as this changes the expected composition of rivals in later rounds conditional on qualification. Moreover, in equilibrium, it may also depend on the chance of elimination of each rival in one of the future rounds. This makes it difficult to derive a general characterization result for contests with equally many qualification spots and prizes in multiple periods.

Thus, in the second part of the paper, we focus on contests with two rounds, two prizes in Round 1 and two qualification spots. In Round 2, the two remaining players compete for a 
single prize. We provide a closed-form characterization of a subgame-perfect equilibrium in Proposition 3. We then further restrict attention to two polar situations where two players have the same valuation. This approach allows us to disentangle effects of externality payoffs and qualification payoffs, as in each case one of the two vanishes. Moreover, we provide uniqueness results for this setting and we use it for our comparisons to other models.

As a first benchmark for comparison, we consider a static contest in which, as in the twostage contest, the winner receives two prizes and the contestant with the second highest effort gets one prize. We show that the dynamic model yields a higher expected effort. As a second benchmark, we compare our results to a version in which the second round is not announced ex-ante by the contest designer. For this case, we identify when the externality payoff yields an extra motivation in terms of expected effort. We see our results as a first indication that extra incentives through the newly explored externality payoffs are an explanation for the popularity of the studied class of contests.

The first part of this paper is closely related to the classic literature on static all-pay auctions. Inspired by the pioneering work by Hillman and Samet (1987) and Hillman and Riley (1989), Baye, Kovenock, and de Vries (1996) provided a complete characterization of equilibria in the complete-information all-pay auction with one prize. Under a weak genericity condition, their characterization was extended to all-pay auctions with multiple identical prizes in Clark and Riis (1998) and even more general all-pay contests with multiple identical prizes in Siegel (2009).

The second part of the paper relates to the recent literature on identity-dependent externalities in all-pay auctions with a single prize; see Konrad (2006), Klose and Kovenock (2015a) and Klose and Kovenock (2015b). In these papers, if an agent does not win a prize, his utility depends on the identity of the winner. For example, in a political campaign, the utility of a losing party might depend on its ideological difference to the winner. In our paper, however, the payoff of a player in a given round does not depend the identities of the (other) winners. Instead, the effort decision influences the composition of the rivals in future rounds, and thereby the expected future payoff. Thus, the size of the externality 
is endogenously determined. Moreover, the externality effect in our paper depends on the entire set of qualifiers and not only on the winner.

Finally, our model is related to literature on multi-stage contests/races (see, e.g., Harris and Vickers, 1987, and with the all-pay auction as a stage game, Konrad and Kovenock, 2009). As a main difference to a race, the set of players shrinks over time in our model. Moreover, races do not have a fixed time horizon, but end once a player reaches a certain target or decides to stop.

We proceed as follows. Section 2 sets up the model. In Sections 3 and 4, we present the main characterization results. Section 5 compares our results to two benchmark models. Section 6 discusses our findings. The proofs are relegated to the appendix.

\section{The Model}

Consider a model with $n>2$ risk-neutral contestants $i \in\{1,2, \ldots, n\}=I$ and $T>1$ rounds $t \in\{1,2, \ldots, T\}$. All $n$ contestants are qualified for Round 1 . We denote the set of contestants which are qualified for Round $t$ by $Q_{t-1}$. Note that $Q_{0}=I$. Furthermore, denote the number of bidders qualified for Round $t$ by $q_{t-1}=\left|Q_{t-1}\right|$. At time $t$, each contestant has complete information about the entire history of bids/eliminations up to time $t .^{3}$ Each qualified contestant $i \in Q_{t-1}$ chooses his bid $x_{i t}$ in Round $t$ at a cost of $c\left(x_{i t}\right)=x_{i t} \cdot{ }^{4}$ This yields a vector of bids $x_{t}=\left(x_{i t}\right)_{i \in Q_{t-1}}$.

If all bids are of different size, we say that the player with the highest bid has bid rank 1 , the player with the second highest bid has bid rank 2 and so forth. In Round $t$, the $q_{t}$ bidders with the lowest bid ranks qualify for Round $t+1$. The $m_{t} \leq q_{t}$ players with the lowest bid ranks in Round $t$ additionally receive a prize, where $m_{t} \geq 0$. To rule out trivial cases where nothing is at stake in the final round, we require $m_{T} \geq 1$ and $m_{T}<q_{T-1}$.

\footnotetext{
${ }^{3}$ The equilibrium strategies depend only on the identities of the other qualifiers. Hence, our results would also hold when only the identities of the qualifiers and their valuations are observable.

${ }^{4} \mathrm{As}$ is well-known in this literature, the valuation-cost ratio determines the effort levels. We thus normalize individual marginal costs to one and consider the differences only in the valuations. Our equilibrium characterization directly extends to asymmetric, yet constant marginal costs.
} 
The $q_{t-1}-q_{t} \geq 0$ players with the highest bid ranks are eliminated. Thus, the vector $x_{t}$ splits the players into three mutually exclusive sets: (i) players who receive a prize and qualify for the next round, (ii) players who do not receive a prize and qualify for the next round and (iii) players who do not receive a prize and are eliminated, i.e., do not qualify for the next round. Each stage has at least two contestants, i.e., $q_{T-1} \geq 2$. We employ the convention $q_{T}=q_{T-1}$.

If at least two players bid the same amount, a tie-breaking rule specifies the bid rank among players involved in a tie. We now define one possible tie-breaking rule. The only result in the paper that depends on this specification is Part (1) of Proposition 2. Part (2) of Proposition 2 contains a general result for arbitrary tie-breaking rules. The bid rank of player $i$ in Round $t$ is given by

$$
r_{i t}\left(x_{t}\right)=\left|j \in Q_{t-1}: x_{j t}>x_{i t}\right|+\mid j \in Q_{t-1}: x_{j t}=x_{i t} \text { and } j \leq i \mid .
$$

The second term on the right-hand side of Eq. (1) is at least 1 due to the case $j=i$. Note that Eq. (1) formalizes the previous description when all bids are of different size. Moreover, it entails that ties are broken in favor of players with lower subscripts.

Valuations for a prize in Round $t$ are denoted by $v_{i t}$, where $v_{i t}=0$ if $m_{t}=0$ and $v_{i t}>0$ otherwise for all $i$ and $t$. For each player $i$, his (mixed) strategy specifies a cumulative distribution (cdf) $F_{i t}$ over his bids for every history such that player $i$ is qualified for Round $t$. A player is called active in Round $t$ if he is qualified for Round $t$ and $F_{i t}(0) \neq 1$. Given the distributions of the other players, the (expected) payoff of a qualified player in Round $t$ who bids $x_{i t}$ is $\pi_{i t}\left(x_{i t}\right)=v_{i t} \mathbb{P}\left[\left\{r_{i t}\left(x_{t}\right) \leq m_{t}\right\} \mid x_{i t}\right]-x_{i t}$. If player $i$ did not qualify for Round $t, \pi_{i t}=0$. Each player maximizes his expected payoff $\pi_{i}=\mathbb{E}\left[\sum_{t=1}^{T} \pi_{i t}\right]$.

\section{General Results}

In several applications, all players who qualify for the next round receive a prize. For instance, all participants gain some marketing revenues and/or derive intrinsic utility from the mere qualification for a World Championship or Olympic Games. In this example, 
different valuations might reflect differences in the marketability of the athlete or different effort costs. In other applications, however, there are fewer prizes than qualification spots, e.g., when only the winner of a preliminary round receives an additional intermediate prize. We shall see that this distinction has a qualitative impact on the equilibrium characterization.

We first recall the well-known result for static all-pay auctions with different valuations and $m$ identical prizes by Clark and Riis (1998) and adapt it to our notation.

Lemma 1 (Proposition 1 in Clark and Riis, 1998). Consider a static all-pay auction with $m<n$ identical prizes and let the valuations be ordered by $v_{1}>v_{2}>\ldots>v_{n}$. There exists a unique mixed strategy Nash equilibrium of the game in which only the bidders with the $m+1$ highest valuations are active. These players randomize according to the probability distributions $F_{i}(x)$ over $\left[\underline{x}_{i}, v_{m+1}\right]$ with common upper support $\bar{x}_{i}=\bar{x}=v_{m+1}$ and lower supports given by $\underline{x}_{m+1}=0$ and $\underline{x}_{i}=\left[1-\prod_{j=i}^{m}\left(\frac{v_{j}}{v_{i}}\right)\right] v_{m+1}$ for $i=1,2, \ldots, m$ and where

$$
F_{i}(x)=1-\frac{v_{i}}{\prod_{j=k}^{m} v_{j}^{\frac{1}{m+1-k}}}\left(1-\frac{x}{v_{m+1}}\right)^{\frac{1}{m+1-k}}
$$

for $i=1,2, \ldots, m$ where $k=1$ if $\underline{x}_{1} \leq x \leq v_{m+1}, k=s$ if $\underline{x}_{s} \leq x<\underline{x}_{s-1} s=2,3, \ldots, m$ and

$$
F_{m+1}(x)=1-\frac{v_{m+1}}{v_{m}}+\frac{v_{m+1}}{v_{m}}\left(1-\frac{v_{m}}{\prod_{j=k}^{m} v_{j}^{\frac{1}{m+1-k}}}\left(1-\frac{x}{v_{m+1}}\right)^{\frac{1}{m+1-k}}\right) .
$$

The expected net surplus of an active player $i$ is $v_{i}-v_{m+1}$.

For the rest of this section, we impose that the ordering of players in terms of "strength", e.g., cost-efficiency or ability, is the same across periods.

Assumption 1 (Constant Valuation Order Condition). The valuations satisfy $v_{1 t}>v_{2 t}>$ $v_{3 t}>\ldots>v_{n t}$ for all $t$ such that $m_{t}>0$.

To state the first main result, we need some additional notation. Recall that $\left|Q_{t-1}\right|=$ $q_{t-1}$. Define the set $\tilde{Q}_{t-1}=\left\{1,2, \ldots, q_{t-1}\right\}$ and the function $g_{t}: Q_{t-1} \rightarrow \tilde{Q}_{t-1}$ such that $g_{t}(i)=\left|j \in Q_{t-1}: j \leq i\right|$. Thus, the set $\tilde{Q}_{t-1}$ is a relabeling of the qualified players ordered by their valuations. 
Proposition 2. (1) Suppose $m_{t}<q_{t}$ for all $t<T$ and ties are broken as specified in Section 2. The contest has a unique subgame-perfect equilibrium. For any given set of qualified players $Q_{t-1}$, equilibrium behavior in Round $t$ is described as follows. If $m_{t}>0$, equilibrium behavior is given by the construction obtained in Clark and Riis (1998), where $q_{t-1}$ is the number of players, $m_{t}$ is the number of prizes and the valuations satisfy $v_{g_{t}(i)}=v_{i t}$ for all $i \in Q_{t-1}$. If $m_{t}=0$, every player $i \in Q_{t-1}$ bids zero with probability 1 .

(2) Suppose $m_{t}<q_{t}$ for all $t<T$ and $m_{t}$ is decreasing in $t$. Then the result in Part (1) extends to arbitrary tie-breaking rules.

(3) Suppose $m_{s}=q_{s}$ for at least one round $s<T$. Then there exists no subgame-perfect equilibrium in which behavior in all such rounds is given by the construction obtained in Clark and Riis (1998).

Note that in the equilibrium of Clark and Riis (1998) only the players with $m_{t}+1 \leq q_{t}$ highest valuations are active. Thus, due to the relative scarcity of prizes, only the "strongest" contestants find it valuable to compete. In (1) and (2), this means that no strong contestant runs the risk of elimination. Thus, a contest with more qualification spots than prizes provides no extra incentives from qualification.

The assumptions for Parts (1) and (2) are quite restrictive. To recap, we need that (i) the number of qualification spots exceeds the number of prizes, (ii) valuations are ordered in a constant way across periods, and (iii) the prize sequence is decreasing or a special tiebreaking rule is used. For instance, in a sports competition, the qualification to a World Championship or Olympiad is a "prize" given to each qualifying athlete. In this case, a strong athlete has an extra incentive from qualification based on the fact that strong rivals might not qualify which is not taken into account in the one-shot model.

Thus, it seems interesting to study how equilibrium behavior changes if the previous assumptions are not met. For the general model, it is hard to quantify the extra incentive from qualification, as it does not only depend on whether a player qualifies, but also on the other qualifiers: the expected future payoff of a contestant might increase when the other qualifiers are weaker than expected. We analyze a tractable two-period version in Section 4. 
Before doing so, let us briefly elaborate on two extensions of Proposition 2.

Discounting: The proof of Proposition 2 does not use the relative importance of a particular round. Thus, it directly extends to cases in which payoffs in each Round $t$ are discounted at an arbitrary common discount rate $\delta_{t}>0$.

General Cost Functions: The first two parts of Proposition 2 can be extended to more general, non-linear cost functions. A sufficient condition is that the valuation-cost ratio is ordered in the same way for each round and for every bid, an extension of our valuation order condition. Siegel (2009) provides an even weaker condition on the valuation-cost ratios in his Theorem 2. Together with the requirement that the ratios are ordered identically across rounds, it is also sufficient for the first two parts of Proposition 2 to hold.

\section{Two-Period Model}

We now relax the assumption of more qualification spots than prizes. This is natural for our example of qualifying for the Olympic Games, where each qualification spot is perceived as a prize and can generate additional marketing revenues. In that example, in line with our constant valuation order condition, ability advantages are likely to persist across rounds and the prize sequence might well be decreasing. However, note that we could also relax the other assumptions needed for Proposition 2 (1) and (2) and we would obtain similar effects.

As we have seen in the previous section, when there are equally many qualification spots and prizes, some players have extra incentives from qualification for the next round. In this section, we quantify these extra incentives. We henceforth restrict attention to two round contests with two qualification spots and three heterogeneous prizes, two in the first round and one in the second round.

\subsection{Three Different Valuations}

Consider $n>3$ contestants with valuations $v_{1}=v_{11}=v_{12} \geq v_{2}=v_{21}=v_{22} \geq \ldots \geq v_{n}=$ $v_{n 1}=v_{n 2}>0$ and $q_{1}=2, m_{1}=2, m_{2}=1$. In words, there are three homogeneous prizes (two in Round 1 and one in Round 2) and two players qualify for Round 2. 
For the two qualifiers, equilibrium play in Round 2 is given by the equilibrium of an allpay auction with asymmetric valuations (see Hillman and Riley, 1989 - the predecessor to Clark and Riis, 1998 for only one prize). The expected equilibrium payoffs are the difference in valuations for the stronger player and zero for the weaker player. To determine the subgame-perfect equilibrium, we now focus on Round 1. For notational convenience, we henceforth omit the time subscript for Round 1 in the notation of $F, x$ and $v$.

If we suppose that only the three players with the highest valuations are active and that the cdf's are continuous, we can write the expected payoff of player 1 as

$$
\begin{array}{r}
\pi_{1}(x)=v_{1}\left(F_{2}(x)+F_{3}(x)-F_{2}(x) F_{3}(x)\right)-x+ \\
\left(v_{1}-v_{2}\right)\left(F_{2}(x)+F_{3}(x)-F_{2}(x) F_{3}(x)\right)+\left(v_{2}-v_{3}\right)\left(F_{2}(x)\left(1-F_{3}(x)\right)+\int_{0}^{x} f_{3}(z) F_{2}(z) d z\right) .
\end{array}
$$

The first line of the above equation represents the expected payoff of player 1 in Round 1. It is given by her valuation times the probability that she does not have the lowest bid in the first round minus the cost of the bid. The second line expresses the expected payoff in Round 2. The first term is the qualification payoff which gives the direct value of the own qualification. It is given by the qualification probability times the expected payoff $\left(v_{1}-v_{2}\right)$ of competing against player 2 in a one-shot all-pay auction (Round 2). The last term gives externality payoff which is the expected additional payoff from the chance that player 2 is eliminated and player 1 can compete against player 3 in Round 2.

For player 2, the qualification payoff is zero, as if she qualifies with player 1 , she gets no additional expected payoff in Round 2. Thus, we obtain

$$
\pi_{2}(x)=v_{2}\left(F_{1}(x)+F_{3}(x)-F_{1}(x) F_{3}(x)\right)-x+\left(v_{2}-v_{3}\right)\left(F_{1}(x)\left(1-F_{3}(x)\right)+\int_{0}^{x} f_{3}(z) F_{1}(z) d z\right) .
$$

Finally, for player 3, the qualification payoff and the externality payoff are both zero, as he receives an expected payoff of zero in Round 2 against both possible opponents, i.e.,

$$
\pi_{3}(x)=v_{3}\left(F_{1}(x)+F_{2}(x)-F_{1}(x) F_{2}(x)\right)-x .
$$


Before we construct the equilibrium in special cases, we first provide our general characterization result: ${ }^{5}$

Proposition 3. In a subgame-perfect equilibrium, the distribution functions in Round 1 are given by

$$
\begin{aligned}
& F_{1}(x)= \begin{cases}0 & \text { for } x<a \\
1-\left(1-\frac{x}{v_{3}}\right)^{\frac{1}{2}}\left(1-\frac{a}{v_{3}}\right)^{-\frac{1}{2}} & \text { for } x \in\left[a, v_{3}\right] \\
1 & \text { for } x>v_{3},\end{cases} \\
& F_{2}(x)= \begin{cases}\frac{x}{v_{3}} & \text { for } x<a \\
1-\left(1-\frac{a}{v_{3}}\right)^{\frac{1}{2}}\left(1-\frac{x}{v_{3}}\right)^{\frac{1}{2}} & \text { for } x \in\left[a, v_{3}\right] \\
1 & \text { for } x>v_{3},\end{cases}
\end{aligned}
$$

and

$$
F_{3}(x)= \begin{cases}b+\frac{x}{v_{2}} & \text { for } x<a \\ 1-\left(1-b-\frac{a}{v_{2}}\right)\left(1-\frac{a}{v_{3}}\right)^{-\frac{1}{2}}\left(1-\frac{x}{v_{3}}\right)^{\frac{1}{2}} & \text { for } x \in\left[a, v_{3}\right] \\ 1 & \text { for } x>v_{3},\end{cases}
$$

where $a=\frac{4 v_{3}\left(v_{1}-v_{2}\right)}{4 v_{1}-v_{2}-v_{3}}$ and $b=\frac{\left(v_{2}-v_{3}\right)\left(4 v_{1}-v_{2}\right)}{v_{2}\left(4 v_{1}-v_{2}-v_{3}\right)}$.

All other players bid 0 with probability 1 . Among the qualified contestants, the distribution functions in Round 2 are as given in Hillman and Riley (1989).

We now consider the two limiting cases where two valuations are equal. This enables us to separate effects induced by the qualification payoff and by the externality payoff. In addition, we provide more insights into the equilibrium construction and uniqueness.

\footnotetext{
${ }^{5}$ We are not aware of the existence of another subgame-perfect equilibrium. However, as in the setting of Klose and Kovenock (2015a), general uniqueness results are difficult to obtain due to the externalities.
} 


\subsection{Two Strong Players and One Weak Player}

In the first case, valuations are $v_{1}=v_{11}=v_{12}=v_{2}=v_{21}=v_{22}>v_{3}=v_{31}=v_{32}>0$ and $q_{1}=2, m_{1}=2, m_{2}=1$. Hence, two players with equal valuations compete with one "weaker" player. As before, the two qualified players play the one-shot equilibrium of an all-pay auction in Round 2. It remains to analyze the first round.

Define the left endpoint of the support of the cdf of player $i$ by $\underline{x}_{i}=\left\{\inf x: F_{i}(x)>0\right\}$ and the left endpoint of the support of the joint cdf by $\underline{x}=\min _{i}\left\{\underline{x}_{i}\right\}$. Analogously, for the right endpoint, define $\bar{x}_{i}=\left\{\sup x: F_{i}(x)<1\right\}$ and $\bar{x}=\max _{i}\left\{\bar{x}_{i}\right\}$. In this subsection, we focus on equilibria in the following class of strategy profiles.

Definition 1. A strategy profile is symmetric if $F_{1}=F_{2}$. A strategy profile is monotone if for all $i, F_{i}(x)$ has a strictly positive density on $\left(\underline{x}_{i}, \bar{x}_{i}\right)$.

Symmetric and monotone equilibria are a natural subclass of equilibria. Symmetry requires that ex-ante identical contestants behave in the same way. Monotonicity mainly requires that there are no "holes" in the support of a cdf. In a model with vanishing perturbations in mapping effort to output, Seel and Strack (2016) show that any cdf has to satisfy this property.

By a standard undercutting argument, each distribution function $F_{i}$ is continuous on $\left(\underline{x}_{i}, \bar{x}_{i}\right)$. The following lemma is also similar to well-known results in the related literature on all-pay auctions.

Lemma 4. The endpoints are given by $\underline{x}_{1}=\underline{x}_{2}=\underline{x}_{3}=0$ and $\bar{x}_{1}=\bar{x}_{2}=\bar{x}_{3}=v_{3}$. Moreover, we have $F_{1}(0)=F_{2}(0)=0$ and $\pi_{3}=0$.

By monotonicity, each player is indifferent between any bid in $(0, \bar{x})$. Since the cdf's are continuous, the payoff of player 3 on $(0, \bar{x})$ can be written as

$$
\pi_{3}(x)=v_{3}\left(F_{1}(x)+F_{2}(x)-F_{1}(x) F_{2}(x)\right)-x=0 .
$$

Imposing symmetry in Eq. (2) and rearranging, we obtain

$$
F_{1}(x)=F_{2}(x)=1-\sqrt{1-\frac{x}{v_{3}}} .
$$


The payoff of player 1 on $(0, \bar{x})$ can be written as $\pi_{1}(x)=v_{1}\left(F_{2}(x)+F_{3}(x)-F_{2}(x) F_{3}(x)\right)-x+\left(v_{1}-v_{3}\right)\left(F_{2}(x)\left(1-F_{3}(x)\right)+\int_{0}^{x} f_{3}(z) F_{2}(z) d z\right)$.

As in the general case, the first two terms represent the expected payoff of player 1 in Round 1. However, the qualification payoff is now zero as player 1 does not get a positive payoff if she qualifies to Round 2 with an equally strong player. Thus, we only have the externality payoff for Round 2 .

Plugging in for $F_{2}(x)$ and taking the derivative, we obtain the condition under which player 1 is indifferent between her bids as the differential equation

$$
v_{1}\left(1-\frac{x}{v_{3}}\right) f_{3}(x)=\frac{2 v_{1}-v_{3}}{2 v_{3}} F_{3}(x)+\sqrt{1-\frac{x}{v_{3}}}-\frac{2 v_{1}-v_{3}}{2 v_{3}}
$$

subject to the boundary condition $F_{3}\left(v_{3}\right)=1$.

The solution is given by

$$
F_{3}(x)=1-\frac{2 v_{3} \sqrt{1-\frac{x}{v_{3}}}}{3 v_{1}-v_{3}} .
$$

Note that we obtain the same equilibrium distribution as in the limit when $v_{2}$ approaches $v_{1}$ in the general case. The following proposition sums up the previous findings.

Proposition 5. The game has a unique symmetric and monotone subgame-perfect equilibrium. In this equilibrium, the distribution functions for the bids in Round 1 are

$$
\begin{gathered}
F_{1}(x)=F_{2}(x)= \begin{cases}1-\left(1-\frac{x}{v_{3}}\right)^{\frac{1}{2}} & \text { for } x \in\left[0, v_{3}\right] \\
1 & \text { for } x>v_{3},\end{cases} \\
F_{3}(x)= \begin{cases}1-\frac{2 v_{3}\left(1-\frac{x}{v_{3}}\right)^{\frac{1}{2}}}{3 v_{1}-v_{3}} & \text { for } x \in\left[0, v_{3}\right] \\
1 & \text { for } x>v_{3},\end{cases}
\end{gathered}
$$

Among the qualified contestants, the distribution functions in Round 2 are as given in Hillman and Riley (1989). 

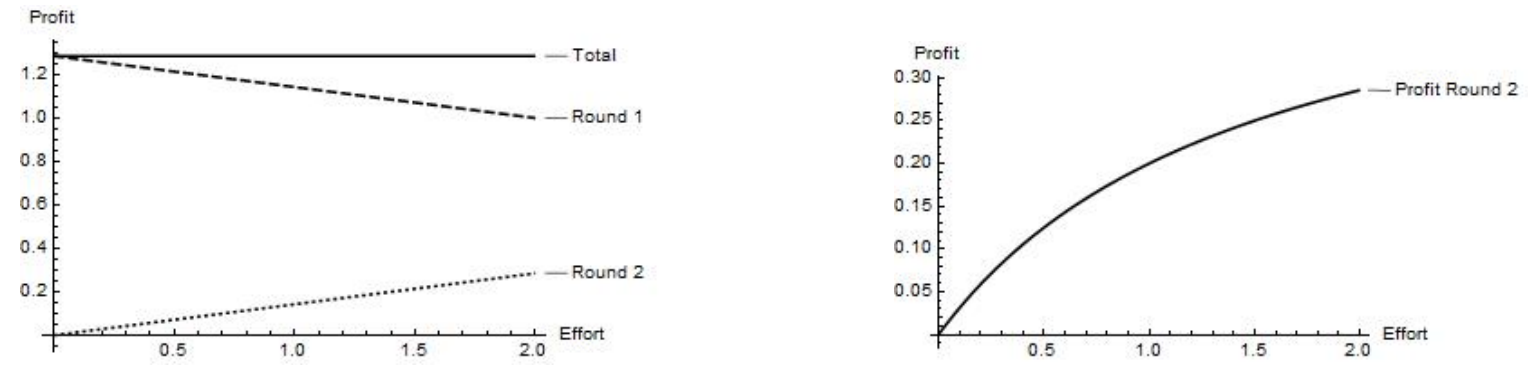

Figure 1 Both panels show the bid of player 1 in Round 1 on the $\mathrm{x}$-axis and her profit on the $\mathrm{y}$-axis for valuations $v_{1}=v_{2}=3$ and $v_{3}=2$. The left panel shows the expected profit per round. The right panel shows her expected externality payoff in Round 2 conditional on qualification with a given bid in Round 1.

The expected profit of player 1 is displayed for both rounds in the left panel of Figure 1. The total profit is constant as, in equilibrium, player 1 randomizes her bids over the entire interval. Her expected payoff in the first round, however, is higher at lower effort levels. Intuitively, as the effort approaches zero, if she qualifies, she will almost always face player 2 in Round 2. In this case, she receives no additional payoff in Round 2, i.e., her qualification payoff is zero. On the other hand, for higher bids, the probability of qualifying for Round 2 together with player 3, i.e., her externality payoff, increases. This increase is offset by a lower expected payoff in Round 1.

The right panel illustrates the payoff of player 1 in Round 2 for a given bid in Round 1 conditional on qualifying for Round 2 with that bid. Again, we can see that a higher bid in Round 1 leads to a higher externality payoff caused by an increased likelihood of qualifying together with player 3 .

\subsection{Two Weak Players and One Strong Player}

The next example deals with 3 contestants with valuations $v_{1}=v_{11}=v_{12}>v_{2}=v_{21}=$ $v_{22}=v_{3}=v_{31}=v_{32}>0$ and $q_{1}=2, m_{1}=2, m_{2}=1$. Thus, there is only one "strong" player and two "weak" players. 
Among the two qualified contestants, equilibrium play in Round 2 is given by the equilibrium in Hillman and Riley (1989). In particular, note that the expected payoff in Round 2 is zero for players 2 and 3 independent of the identity of their rival. The expected payoff of player 1 is $v_{1}-v_{3}$ in Round 2, again independent of the identity of the rival. Note that this means, that all externality payoffs are zero and the qualification payoff for player 1 is maximal. ${ }^{6}$

The payoffs in the first round are equivalent to those in a static game with two prizes and valuations $2 v_{1}-v_{3}$ for the strong player and $v_{3}$ for the weak players. The equilibrium can be obtained by plugging these valuations in the equilibrium found in Clark and Riis (1998). After some rearranging, we obtain:

Proposition 6. In the unique subgame-perfect equilibrium, the distribution functions in Round 1 are given by

$$
F_{1}(x)= \begin{cases}0 & \text { for } x<v_{3}\left(1-\frac{v_{3}}{2 v_{1}-v_{3}}\right) \\ 1-\left(1-\frac{x}{v_{3}}\right)^{\frac{1}{2}}\left(\frac{2 v_{1}-v_{3}}{v_{3}}\right)^{\frac{1}{2}} & \text { for } x \in\left[v_{3}\left(1-\frac{v_{3}}{2 v_{1}-v_{3}}\right), v_{3}\right] \\ 1 & \text { for } x>v_{3},\end{cases}
$$

and

$$
F_{2}(x)=F_{3}(x)= \begin{cases}\frac{x}{v_{3}} & \text { for } x<v_{3}\left(1-\frac{v_{3}}{2 v_{1}-v_{3}}\right) \\ 1-\left(1-\frac{x}{v_{3}}\right)^{\frac{1}{2}}\left(\frac{v_{3}}{2 v_{1}-v_{3}}\right)^{\frac{1}{2}} & \text { for } x \in\left[v_{3}\left(1-\frac{v_{3}}{2 v_{1}-v_{3}}\right), v_{3}\right] \\ 1 & \text { for } x>v_{3} .\end{cases}
$$

Among the qualified contestants, the distribution functions in Round 2 are as given in Hillman and Riley (1989).

\footnotetext{
${ }^{6}$ The existence of an externality payoff is linked to the heterogeneity of the contestants. In particular, Assumption 1 does not hold in this section as $v_{2}=v_{3}$.
} 


\section{Comparisons to Related Contests}

In the previous section, we derived equilibria for cases in which the winner of Round 2 collects 2 identical prizes, the loser of Round 2 receives 1 prize and the player who is eliminated in Round 1 receives no prize.

\subsection{Static Contest}

The corresponding static model is a multi-prize all-pay auction with prize $2 v_{i}$ for the highest bidder and $v_{i}$ for the second highest bidder. As before, players are ordered by their valuation, i.e., $v_{1} \geq v_{2} \geq v_{3}$. In the appendix, we verify the conditions for applicability of the algorithm in Xiao (2016) and use it to derive the unique equilibrium of the static model. Then, we compare the induced effort levels in both contests. We focus on the polar cases with two equal valuations, as these yield a unique (symmetric and monotone) equilibrium in the two-stage contest and are thus suitable for comparative statics.

Proposition 7. For both cases $\left(v_{1}>v_{2}=v_{3}\right.$ and $\left.v_{1}=v_{2}>v_{3}\right)$, the total expected effort in the two-stage contest is higher than in the static contest.

The intuition for Proposition 7 differs for the two cases. In the case of two strong contestants and one weak contestant (see Figure 2), the static contest might yield very low effort provision if valuations differ drastically. In particular, there are cases where the third player remains inactive and the other two contestants essentially compete over one prize as they are guaranteed second place. Instead, they compete for two prizes in Round 1 of the two-stage intragroup elimination contest and all three players are active.

For two weak contestants and one strong contestant, player 1 is additionally motivated by the qualification payoff. Thus, in the two-stage contest, player 1 exerts relatively more effort. In turn, this leads to a higher total equilibrium effort. 


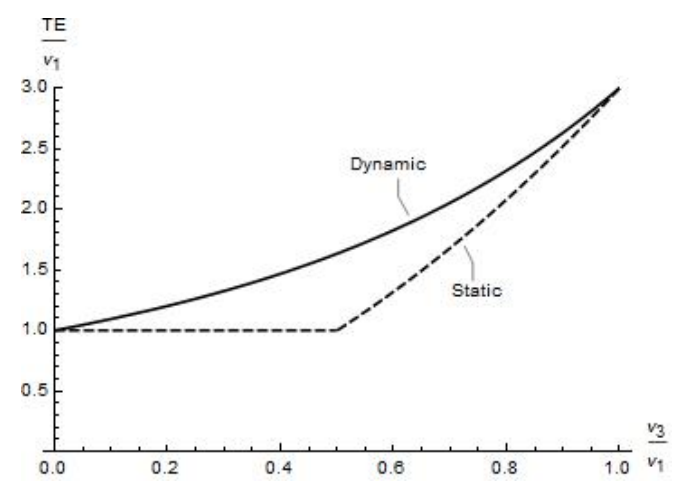

Figure 2 The $\mathrm{x}$-axis depicts the fraction of valuations $\frac{v_{3}}{v_{1}}$ and the $\mathrm{y}$-axis the total effort normalized by $v_{1}$. The solid line depicts the expected total effort in the two-stage contest and the dotted line the expected total effort in the static game in the case $v_{1}=v_{2}>v_{3}$.

\subsection{No Announcement of Second Round}

In many applications, a designer knows whether she will host a similar contest in the future. If so, she has the option to reveal it and announce that the best performers will be invited again or to keep this information private. We assume that contestants do not anticipate further rounds if the designer does not announce them. ${ }^{7}$ Then the question becomes: Should the designer announce future rounds?

Without announcement, the equilibria in Clark and Riis (1998) will be played, with three players and two prizes in Round 1, and two players and one prize in Round 2. We provide the details in the appendix. The next result compares the two informational assumptions.

Proposition 8. Announcing the second round

(i) increases expected effort if and only if $v_{3}<\frac{\sqrt{17}-3}{2} v_{1}$ for $v_{1}=v_{2}>v_{3}$,

(ii) increases expected effort if and only if $v_{3}<\left(2-\frac{(9-\sqrt{78})^{\frac{1}{3}}}{3^{\frac{2}{3}}}-\frac{1}{(27-3 \sqrt{78})^{\frac{1}{3}}}\right) v_{1}$ for $v_{1}>v_{2}=v_{3}$, (iii) increases the chance that a contestant with the highest valuation wins Round 2 for $v_{1}=v_{2}>v_{3}$ and $v_{1}>v_{2}=v_{3}$.

\footnotetext{
${ }^{7}$ The less extreme case where the designer can only influence the belief that another round follows leads to qualitatively similar results.
} 
The cutoff values in (i) and (ii) are close to $v_{3}=0.5 v_{1}$. Announcing is better in terms of expected effort when there is more dispersion in variations. Let us provide some intuition for the underlying effects. When Round 2 is announced, the stronger players have more to gain in Round 1, which explains (iii). For valuations as given in (ii), total expected effort in Round 1 increases. However, the chance that player 1 qualifies also increases, which reduces expected effort in Round 2 by the exclusion principle (Baye, Kovenock, and de Vries, 1993). For valuations as given in (i), when Round 2 is announced, player 3 reduces her effort in Round 1. In turn, more effort is spent in Round 2, as the case in which players 1 and 2 qualify is more likely to occur.

\section{Discussion}

The main novelty of our analysis has been the focus on the externality payoff, that is the gain by the possibility that strong rivals are eliminated along the way. The first part of the paper has derived sufficient conditions under which an externality payoff has no effect: each round has more qualification spots than prizes, the order of valuation is constant across rounds, and the number of prizes is decreasing.

These assumptions appear to be rather restrictive. Hence, a better understanding of externality payoffs seems important. For analytical tractability, we have focused on cases with two rounds and equal qualification spots and prizes in the first round. For this setting we have been able to obtain closed-form solutions. The expected payoff of qualifying for the second round can be disentangled into the qualification payoff and the externality payoff. The qualification payoff describes the payoff of qualifying with the best competitors. We have analyzed the extreme cases, where only one of the two payoffs is present, and shown that the externality payoff vastly influences equilibrium behavior already in this simple environment.

The comparative statics for these cases might be seen as a first indication why such contests are frequently observed in the real world. We hope to inspire future research on externality payoffs within and beyond the current model framework. 


\section{Appendix}

Proof of Proposition 2: We start with Part (1). Existence: Define $\tilde{Q}_{t-1}^{b}=\{1,2, \ldots, b\} \subseteq$ $\tilde{Q}_{t-1}$, i.e., $b \leq q_{t-1}$. Note that by the equilibrium construction in Clark and Riis (1998), only players $i$ such that $g_{t}(i) \in \tilde{Q}_{t-1}^{m_{t}+1}$ are active in Round $t$. Since $q_{t}>m_{t}$ or equivalently $q_{t} \geq m_{t}+1$, in our equilibrium candidate, player $i$ qualifies for Round $t+1$ if and only if $g_{t}(i) \in \tilde{Q}_{t-1}^{q_{t}}$ (recall that ties of players who bid zero are resolved according to their subscripts).

First consider a deviation by a player $i$ such that $g_{t}(i) \in \tilde{Q}_{t-1}^{q_{t}}$. For any deviation, the other qualifying players and their strategies in the remaining rounds stay the same, i.e., player $i$ 's payoff in later rounds is unchanged. By Lemma 1, player $i$ cannot increase his payoff in Round $t$. Thus, no player $i$ such that $g_{t}(i) \in \tilde{Q}_{t-1}^{q_{t}}$ has an incentive to deviate in Round $t$.

Now consider a deviation by a player $i$ such that $g_{t}(i) \in \tilde{Q}_{t-1} \backslash \tilde{Q}_{t-1}^{q_{t}}$ in Round $t$. By optimality in the one-shot game, any deviation of player $i$ yields a (weakly) negative payoff in Round $t$. Moreover, in case of qualification for Round $s>t$, player $i$ has the lowest subscript in Round $s$ by the constant valuation order condition (Assumption 1). Thus, by the equilibrium construction, player $i$ makes at most zero payoff in any Round $s>t$ since $m_{s}<q_{s}$. Thus, player $i$ has no incentive to deviate in Round $t$. Thus, no player has an incentive to deviate in any round $t$, i.e., the strategy profile from the proposition is a subgame-perfect Nash equilibrium.

Uniqueness: Towards a contradiction, suppose there exists another subgame-perfect equilibrium. Then, there exists a maximal round $\bar{t}$ such that the equilibrium cdf's differ from the ones in Clark and Riis (1998) in some subgame starting in Round $\bar{t}$, but are identical to those in the proposition in all subgames starting in any Round $t>\bar{t}$. Consider a subgame starting in Round $\bar{t}$ in which cdf's differ from the ones in the proposition.

Case 1: Suppose no player $i$ such that $f_{\bar{t}}(i) \in \tilde{Q}_{\bar{t}-1} \backslash \tilde{Q}_{\bar{t}-1}^{q_{t}}$ is active in Round $\bar{t}$. Then only the players $i$ such that $f_{\bar{t}}(i) \in \tilde{Q}_{\bar{t}-1}^{q_{t}}$ qualify for the next round. If one of these players deviates in Round $\bar{t}$, the qualifiers for $\bar{t}+1$ and future equilibrium behavior is unchanged. 
Thus, only incentives in Round $\bar{t}$ matter for the profitability of a deviation of one of these players. Since the strategy profile is not the unique Nash equilibrium of the one-shot game by assumption, there exists a profitable deviation of a player $i$ in Round $\bar{t}$, which yields a contradiction to optimality.

Case 2: Complementing Case 1, suppose at least one player $i$ such that $f_{\bar{t}}(i) \in \tilde{Q}_{\bar{t}-1} \backslash \tilde{Q}_{\bar{t}-1}^{q_{t}}$ is active in Round $\bar{t}$. Let $h$ be the active player with the highest subscript. Note that in every Round $t>\bar{t}$, if player $h$ qualifies, he is the remaining player with the lowest valuation by Assumption 1. Since $m_{t}<q_{t}$, by the construction in Clark and Riis (1998)—which holds for all $t>\bar{t}$ by assumption-player $h$ receives an expected payoff of 0 in all Rounds $t>\bar{t}$. Thus, by optimality, $\pi_{h \bar{t}}(x) \geq 0$ for all $x$ in the support of his cdf in Round $\bar{t}$. In particular, the inequality has to hold at the supremum $\bar{x}_{h \bar{t}}$ of his support. Note that $\pi_{h \bar{t}}\left(\bar{x}_{h \bar{t}}\right) \geq 0$ implies $\pi_{j \bar{t}}\left(\bar{x}_{h \bar{t}}\right)>0$ for all $j<h$ such that $j \in Q_{t-1}$. Recall that a player can guarantee a payoff of zero in each round by bidding zero. Thus, the expected continuation payoff satisfies $\mathbb{E}\left[\sum_{\bar{t}}^{T} \pi_{j t}\right]>0$ for all $j<h$ such that $j \in Q_{t-1}$. We again split up the rest of the argument into two cases.

Case 2.1: Suppose $F_{h}(0)=0$. If $F_{j}(0)>0$ for at least one player $j<h$, out of these players, the one with the highest subscript would be eliminated with probability 1 when bidding zero. This contradicts optimality, as the expected continuation payoff should be strictly positive. Thus, $F_{j}(0)=0$ for all $j<h$ such that $j \in Q_{t-1}$.

Define the infimum of the support of the active players by $\underline{x}_{\bar{t}}=\inf \left\{x: \exists i\right.$ s.t. $g_{t}(i) \leq$ $g_{t}(h)$ and $\left.F_{i}(x)>0\right\}$. If $F_{i}\left(\underline{x}_{\bar{t}}\right)>0$ for at least one player $i$ such that $g_{t}(i) \leq g_{t}(h)$, the player with the highest subscript among them is strictly worse off by bidding $\underline{x}_{\bar{t}}$ compared to bidding zero which contradicts optimality. Otherwise, the qualification probability is zero for each player who bids $\underline{x}_{\bar{t}}$. As the continuation payoff is bounded and the winning probability is continuous at $\underline{x}_{\bar{t}}$ in this case, there exists an $\epsilon$-interval such that expected continuation payoffs of bidding $x \in\left[\underline{x}_{\bar{t}}, \underline{x}_{\bar{t}}+\epsilon\right)$ is negative for each player, thereby contradicting optimality.

Case 2.2: Suppose $0<F_{h}(0)<1$. Suppose at least one player $j<h$ has $F_{j}(0)>0$ and call the player with the highest subscript among them player $l$. By optimality of bidding 
zero, the continuation payoff of player $l$ is strictly positive. He never wins a prize in Round $\bar{t}$ by bidding 0 and he can only qualify if only player $h$ is eliminated. In this case, his expected payoff in each round $t \in\{\bar{t}+1, \bar{t}+2, \ldots, T\}$ is weakly lower than if any other rival is eliminated in Round $\bar{t}$ by the construction in Clark and Riis (1998). Note that player $h$ is indifferent between 0 and the supremum of his bids $\bar{x}_{\bar{t}}^{h}$. Thus, player $l$ increases his payoff in Round $\bar{t}$ by deviating from 0 to $\bar{x}_{\bar{t}}^{h}$ and his expected payoff in rounds $t \in\{\bar{t}+1, \bar{t}+2, \ldots, T\}$ also increases weakly. This contradicts optimality of bidding zero.

Thus, $F_{j}(0)=0$ for all qualified players $j<h$. Since their expected payoffs are continuous at 0 , there exists an $\epsilon>0$ such that $F_{j}(x)=0$ for all $x \in[0, \epsilon)$. Extending the definition in Case 2.1, we define the infimum over the positive bids as $\underline{x}_{\bar{t}}=\inf \left\{x: \exists i\right.$ s.t. $g_{t}(i)<$ $g_{t}(h)$ and $F_{i}(x)>0$ or $\left.F_{h}(x)>F_{h}(0)\right\}$. Thus, the qualification probability and the set of qualifiers is constant if a player $j<h$ bids on $\left[0, \underline{x}_{\bar{t}}\right)$. Thus, as in Case 2.1., if at least one player bids $\underline{x}_{\bar{t}}$ with positive probability, one of these players does not improve his winning probability in Round $\underline{x}_{\bar{t}}$ compared to a bid of zero. Moreover, the set of qualifiers remains the same which is a contradiction to optimality of bidding $\underline{x}_{\bar{t}}$. If no bidder bids $\underline{x}_{\bar{t}}$ with positive probability, there is an $\epsilon$-interval such that expected continuation payoffs of bidding $x \in\left[\underline{x}_{\bar{t}}, \underline{x}_{\bar{t}}+\epsilon\right)$ is weakly below the expected continuation payoff of bidding zero for each player, thereby contradicting optimality.

Part (2): Note that on the equilibrium path, the strongest $m_{t}$ contestants qualify with probability one for the next round. Unlike in Part (1), however, the remaining qualifiers (who all bid 0 in Round t) depend on the tie-breaking rule. We can then make a similar case distinction for a possible deviation, but now have to consider players who qualify with probability 1 and players who do not qualify with probability 1. For the former group, the proof stays the same. For the latter group (players who do not qualify with probability 1), by the assumption that $m_{t}$ is decreasing, the behavior of the rivals in future rounds if a different weak contestant qualifies. Thus, such a player cannot make a profit in any future Round and therefore has no incentive to deviate in Round $t$.

It remains to prove Part (3). 
Consider a subgame starting at a Round $s$ such that $m_{s}=q_{s}$ and $m_{t}<q_{t}$ for all $t>s$. Note that $s<T$ since $m_{T}<q_{T}$. Towards a contradiction, suppose that there exists a subgame-perfect equilibrium with behavior in Round $s$ described by Clark and Riis (1998). By the proof of (1), from Round $s+1$ onwards, equilibrium play is given by Clark and Riis (1998). The payoff of the qualified player with the lowest subscript in Period $s$ is constant for all her bids.

Compare two bids in her support in Period $s$. If she qualifies for Period $s+1$ with both bids, her payoff in the remaining rounds is the same. If she qualifies only with the higher bid, she has a positive payoff in the remaining rounds if and only if she chooses the higher bid.

Thus, with a higher bid she makes a higher expected payoff in future rounds and the same expected payoff in the current round $s$, thereby contradicting optimality of the randomization in Clark and Riis (1998) for the subgame starting in Period $s$.

Proof of Proposition 3. We have to show that, given the other player's cdf's, the payoffs are constant on $\left[0, v_{3}\right]$ for players 2 and 3 . Moreover, for player 1 , the payoff has to be constant on $x \in\left[a, v_{3}\right]$ and (weakly) lower for $x<a$ and the payoff of the remaining players cannot be positive for $x>0$. Note that the payoffs of the active players are given by:

$$
\begin{gathered}
\pi_{1}(x)=\left(2 v_{1}-v_{2}\right)\left(F_{2}(x)+F_{3}(x)-F_{2}(x) F_{3}(x)\right)-x+\left(v_{2}-v_{3}\right)\left(F_{2}(x)\left(1-F_{3}(x)\right)+\int_{0}^{x} f_{3}(z) F_{2}(z) d z\right) . \\
\pi_{2}(x)=v_{2}\left(F_{1}(x)+F_{3}(x)-F_{1}(x) F_{3}(x)\right)-x+\left(v_{2}-v_{3}\right)\left(F_{1}(x)\left(1-F_{3}(x)\right)+\int_{0}^{x} f_{3}(z) F_{1}(z) d z\right) . \\
\pi_{3}(x)=v_{3}\left(F_{1}(x)+F_{2}(x)-F_{1}(x) F_{2}(x)\right)-x .
\end{gathered}
$$

We first consider the case $x \in\left[a, v_{3}\right]$ and we plug the distribution functions into the payoffs. We start with player 2 to obtain 


$$
\begin{array}{r}
\pi_{2}(x)=v_{2}\left(1-\left(1-b-\frac{a}{v_{2}}\right)\left(1-\frac{a}{v_{3}}\right)^{-1}\left(1-\frac{x}{v_{3}}\right)\right)-x+\left(v_{2}-v_{3}\right)\left(\left(1-\left(1-\frac{a}{v_{3}}\right)^{-\frac{1}{2}}\right.\right. \\
\left.\left(1-\frac{x}{v_{3}}\right)^{\frac{1}{2}}\right)\left(\left(1-b-\frac{a}{v_{2}}\right)\left(1-\frac{a}{v_{3}}\right)^{-\frac{1}{2}}\left(1-\frac{x}{v_{3}}\right)^{\frac{1}{2}}\right)+\int_{0}^{x} \frac{1}{2 v_{3}}\left(1-b-\frac{a}{v_{2}}\right)\left(1-\frac{a}{v_{3}}\right)^{-\frac{1}{2}} \\
\left(1-\frac{z}{v_{3}}\right)^{-\frac{1}{2}}\left(1-\left(1-\frac{a}{v_{3}}\right)^{-\frac{1}{2}}\left(1-\frac{z}{v_{3}}\right)^{\frac{1}{2}} d z\right.
\end{array}
$$

Further simplification yields

$$
\begin{array}{r}
\pi_{2}(x)=v_{2}\left(1-\left(1-b-\frac{a}{v_{2}}\right)\left(1-\frac{a}{v_{3}}\right)^{-1}\left(1-\frac{x}{v_{3}}\right)\right)-x \\
+\left(v_{2}-v_{3}\right)\left(-\left(1-b-\frac{a}{v_{2}}\right)\left(1-\frac{a}{v_{3}}\right)^{-1}\left(1-\frac{x}{v_{3}}\right)-\frac{x}{2 v_{3}}\right. \\
\left.\left(1-b-\frac{a}{v_{2}}\right)\left(1-\frac{a}{v_{3}}\right)^{-1}+\left(1-b-\frac{a}{v_{2}}\right)+\frac{a}{2 v_{3}}\left(1-b-\frac{a}{v_{2}}\right)\left(1-\frac{a}{v_{3}}\right)^{-1}\right) .
\end{array}
$$

Note that the following relations hold:

$$
\left(1-b-\frac{a}{v_{2}}\right)=-\frac{2 v_{3}}{v_{3}+v_{2}-4 v_{1}} \text { and }\left(1-\frac{a}{v_{3}}\right)^{-1}=\frac{v_{3}+v_{2}-4 v_{1}}{v_{3}-3 v_{2}} .
$$

Consider only terms that involve an $x$ in the expression for $\pi_{2}(x)$ to get

$$
-x+\frac{v_{2}}{v_{3}} x\left(1-b-\frac{a}{v_{2}}\right)\left(1-\frac{a}{v_{3}}\right)^{-1}+\left(v_{2}-v_{3}\right)\left(1-b-\frac{a}{v_{2}}\right)\left(1-\frac{a}{v_{3}}\right)^{-1} \frac{x}{2 v_{3}} .
$$

Note that $x$ vanishes from the expression above by plugging in the two identities from before.

We get the following remainder:

$$
\pi_{2}(x)=v_{2}\left(1+\frac{2 v_{3}}{v_{3}-3 v_{2}}\right)+\left(v_{2}-v_{3}\right)\left(\frac{2 v_{3}}{v_{3}-3 v_{2}}-\frac{2 v_{3}}{v_{2}+v_{3}-4 v_{1}}-\frac{a}{v_{3}-v_{2}}\right) .
$$

Upon simplification, we arrive at the final expression

$$
\pi_{2}(x)=\frac{\left(v_{2}-v_{3}\right)\left(v_{2}-4 v_{1}\right)}{\left(v_{3}+v_{2}-4 v_{1}\right)} .
$$

Similarly, we can consider Player 1 and plug $F_{2}$ and $F_{3}$ into the expression for player 1's payoff: 


$$
\begin{array}{r}
\left(2 v_{1}-v_{2}\right)\left(1-\left(1-b-\frac{a}{v_{2}}\right)\left(1-\frac{x}{v_{3}}\right)\right)-x+\left(v_{2}-v_{3}\right) \\
\left(\frac{1}{2} \frac{a^{2}}{v_{2} v_{3}}+\int_{a}^{x} \frac{1}{2 v_{3}}\left(1-b-\frac{a}{v_{2}}\right)\left(1-\frac{a}{v_{3}}\right)^{-\frac{1}{2}}\right. \\
\left(1-\frac{z}{v_{3}}\right)^{-\frac{1}{2}}\left(1-\left(1-\frac{a}{v_{3}}\right)^{\frac{1}{2}}\left(1-\frac{z}{v_{3}}\right)^{\frac{1}{2}}\right) d z \\
\left.+\left(1-b-\frac{a}{v_{2}}\right)\left(1-\frac{a}{v_{3}}\right)^{-\frac{1}{2}}\left(1-\frac{x}{v_{3}}\right)^{\frac{1}{2}}-\left(1-b-\frac{a}{v_{2}}\right)\left(1-\frac{x}{v_{3}}\right)\right) .
\end{array}
$$

Solving the integral and rearranging, we obtain

$$
\begin{array}{r}
\left(2 v_{1}-v_{2}\right)\left(1-\left(1-b-\frac{a}{v_{2}}\right)\left(1-\frac{x}{v_{3}}\right)\right)-x+\left(v_{2}-v_{3}\right) \\
\left(\frac{1}{2} \frac{a^{2}}{v_{2} v_{3}}+\left(1-b-\frac{a}{v_{2}}\right) \frac{x}{2 v_{3}}+\left(1-b-\frac{a}{v_{2}}\right) \frac{a}{2 v_{3}}\right) .
\end{array}
$$

The expressions including $x$ are

$$
\left(2 v_{1}-v_{2}\right)\left(1-b-\frac{a}{v_{2}}\right) \frac{x}{v_{3}}-x+\left(v_{2}-v_{3}\right)\left(1-b-\frac{a}{v_{2}}\right) \frac{x}{2 v_{3}} .
$$

Using the identities from above, this expression vanishes and hence there is no $x$ left in the payoff equation

$$
\pi_{1}(x)=\left(2 v_{1}-v_{2}\right)\left(1-\left(1-b-\frac{a}{v_{2}}\right)\right)+\left(v_{2}-v_{3}\right)\left(\frac{1}{2} \frac{a^{2}}{v_{2} v_{3}}+\left(1-b-\frac{a}{v_{2}}\right) \frac{a}{v_{3}} .\right.
$$

Finally, plugging in for $F_{1}$ and $F_{2}$, the payoff of player 3 simplifies to

$$
\pi_{3}(x)=v_{3}\left(1-\left(1-\frac{x}{v_{3}}\right)\right)-x=0 .
$$

We now consider the case $0<x<a$. Clearly, the payoff of players 2 and 3 is constant on that interval and continuous at $a$. Thus, they have no profitable deviation from $F_{2}$ and $F_{3}$, respectively.

For player 1, it remains to show that $\pi_{1}(x) \leq \pi_{1}(a)$.

The profit of player 1 is defined as

$$
\pi_{1}(x)=\left(2 v_{1}-v_{2}\right) F_{3}(x)+\left(2 v_{1}-v_{3}\right) F_{2}(x)\left(1-F_{3}(x)\right)-x+\left(v_{2}-v_{3}\right) \int_{0}^{x} f_{3}(z) F_{2}(z) d z .
$$


For all $x<a$, this reduces to

$$
\pi_{1}(x)=\left(2 v_{1}-v_{2}\right)\left(b+\frac{x}{v_{2}}\right)+\left(2 v_{1}-v_{3}\right)\left(\frac{x}{v_{3}}-\frac{b x}{v_{3}}-\frac{x^{2}}{v_{2} v_{3}}\right)-x+\left(v_{2}-v_{3}\right) \frac{x^{2}}{2 v_{2} v_{3}} .
$$

It suffices to show that $\pi_{1}^{\prime}(x)>0$ for all $x<a$. Taking the first derivative yields

$$
\pi_{1}^{\prime}(x)=\left(2 v_{1}-v_{2}\right)\left(\frac{1}{v_{2}}\right)+\left(2 v_{1}-v_{3}\right)\left(\frac{1}{v_{3}}-\frac{b}{v_{3}}-\frac{2 x}{v_{2} v_{3}}\right)-1+\left(v_{2}-v_{3}\right) \frac{x}{v_{2} v_{3}} .
$$

The first derivative is decreasing in $\mathrm{x}$ as $\pi_{1}^{\prime \prime}(x)=-\left(2 v_{1}-v_{3}\right)\left(\frac{2}{v_{2} v_{3}}\right)+\left(v_{2}-v_{3}\right) \frac{1}{v_{2} v_{3}}<0$.

Hence, $\pi_{1}^{\prime}(x) \geq \pi_{1}^{\prime}(a)$ for all $x<a$. It remains to show that $\pi_{1}^{\prime}(a)>0$. We plug in for $a$ and rewrite to obtain

$$
\pi_{1}^{\prime}(a)=\left(2 v_{1}-v_{2}\right)\left(\frac{1}{v_{2}}+\frac{1}{v_{3}}-\frac{b}{v_{3}}-\frac{2 a}{v_{2} v_{3}}\right)+\left(v_{2}-v_{3}\right)\left(\frac{1}{v_{3}}-\frac{b}{v_{3}}-\frac{a}{v_{2} v_{3}}\right)-1 .
$$

Note that $-\frac{b}{v_{3}}>-\frac{v_{2}-v_{3}}{v_{2} v_{3}}$ and $\left(2 v_{1}-v_{2}\right)(-2 a)>-4 v_{3}\left(v_{1}-v_{2}\right)$. Thus,

$\pi_{1}^{\prime}(a)>\left(2 v_{1}-v_{2}\right)\left(\frac{2}{v_{2}}\right)-\frac{4 v_{3}\left(v_{1}-v_{2}\right)}{v_{2} v_{3}}+\left(v_{2}-v_{3}\right)\left(\frac{1}{v_{2}}-\frac{a}{v_{2} v_{3}}\right)-1=1+\left(v_{2}-v_{3}\right)\left(\frac{v_{3}-a}{v_{2} v_{3}}\right)>1$.

To sum up, the payoff of player 1 increases on the interval $[0, a)$, is continuous at $a$ and constant on $\left[a, v_{3}\right]$. Thus, player 1 has no profitable deviation from $F_{1}$ given $F_{2}$ and $F_{3}$.

Finally, consider any of the possibly remaining players $j>3$. Note that if $j$ deviates and qualifies for the second round, she will not receive any expected payoff as she will have the lowest valuation. We define $\rho_{i}(x)$ as the probability that player $i$ wins a prize in Round 1 (and qualifies for Round 2) with a bid of $x$. Note that $v_{j} \leq v_{3}$ and $\rho_{j}(x) \leq \rho_{3}(x)$ in equilibrium. Hence, $\pi_{j}(x)=v_{j} \rho_{j}(x)-x \leq v_{3} \rho_{3}(x)-x \leq 0$. Therefore, bidding 0 is optimal for player $j$.

Proof of Lemma 4. Step 1: $F_{1}(0)=F_{2}(0)=0$.

If $F_{1}(0)=F_{2}(0)>0$, then player 3 has $F_{3}(0)=0$, as bidding $v_{3} \frac{F_{1}(0)}{3}$ yields a strictly higher payoff than bidding zero. As $F_{3}(0)=0$, player 2 gets a strictly higher payoff by bidding $v_{3} \frac{F_{1}(0)}{3}$ than by bidding zero, a contradiction.

Step 2: $\underline{x}_{1}=\underline{x}_{2}=\underline{x}_{3}=0$.

Clearly, a player can secure a payoff of at least zero by bidding zero. Suppose no player $i$ has $\underline{x}_{i}=0$. If $F_{i}(\underline{x})>0$ for at least one player $i$, a player makes a strictly negative profit 
with a bid $\underline{x}$, a contradiction to optimality. Otherwise, by continuity, there exists an $\epsilon>0$ such that the same applies to every bid in an $\epsilon$-neighborhood $(\underline{x}, \underline{x}+\epsilon)$.

Thus, at least one player has $\underline{x}_{i}=0$. Suppose $\underline{x}_{3}=0$ and $\underline{x}_{1}=\underline{x}_{2}>0$. By optimality for player 3 , he does not bid with probability in $\left(0, \underline{x}_{2}\right)$. By the same argument as before, at least one player would prefer to bid $\frac{\underline{x}_{1}}{2}$ instead of $\underline{x}_{1}$ (or in $\left(\underline{x}_{1}, \underline{x}_{1}+\epsilon\right)$ ). Finally, suppose $\underline{x}_{1}=\underline{x}_{2}=0$ and $\underline{x}_{3}>0$. By continuity at 0 , we have $\pi_{1}=\pi_{2}=0$. By optimality, player 3 never bids above $v_{3}$ and thus bidding $v_{3}$ is a profitable deviation for player 1 .

Step 3: $\pi_{3}=0$

Follows immediately as $F_{1}(0)=F_{2}(0)=0$ and $\underline{x}_{3}=0$.

Step 4: $\bar{x}_{1}=\bar{x}_{2}=\bar{x}_{3}=v_{3}$.

First note that $\bar{x}_{1}=\bar{x}_{2}=\bar{x}_{3}$, since any player who has $\bar{x}_{i}>\min _{j} \bar{x}_{j}$ could deviate by bidding $\frac{\bar{x}_{i}+\bar{x}_{j}}{2}$ otherwise. By the zero profit condition of player 3 (Step 3 ), we have $\bar{x}_{3}=v_{3}$.

Proof of Proposition 6. Note that the equilibrium in Clark and Riis (1998) directly extends to include weak inequalities as long as $v_{m+1} \neq v_{m+2}$. Here, we have only 3 players and $m=2$ prizes, so this condition is satisfied. Plugging into their equilibrium construction, the common upper support is $v_{3}$. The lower support of player 3 is 0 . Moreover, we have $\underline{x}_{2}=\left[1-\prod_{j=2}^{2}\left(\frac{v_{j}}{v_{2}}\right)\right] v_{3}=0$. Recall that the expected profit of player 1 if she wins Round 1 is $2 v_{1}-v_{3}$. Thus, we obtain $\underline{x}_{1}=\left[1-\frac{2 v_{1}-v_{3}}{2 v_{1}-v_{3}} \frac{v_{2}}{2 v_{1}-v_{3}}\right] v_{3}=v_{3}\left(1-\frac{v_{3}}{2 v_{1}-v_{3}}\right)$. Plugging our values into the distributions in Clark and Riis (1998) and rearranging gives the characterization in the proposition.

To prove Proposition 7, we first need to derive the equilibrium in the static case.

Lemma 9 (Equilibrium in the Static Contest). Consider the static contest.

Case 1: If $v_{1}=v_{2} \geq 2 v_{3}$ the unique Nash equilibrium is given by

$$
F_{1}(x)=F_{2}(x)= \begin{cases}\frac{x}{v_{1}} & \text { for } x \leq v_{1} \\ 1 & \text { for } x>v_{1}\end{cases}
$$




$$
F_{3}(x)=1 \text { for } x \geq 0 \text {. }
$$

Case 2: If $v_{1}=v_{2}<2 v_{3}$, the unique Nash equilibrium is given by

$$
F_{1}(x)=F_{2}(x)= \begin{cases}\frac{x}{2 v_{3}} & \text { for } x \leq 2 v_{3} \\ 1 & \text { for } x>2 v_{3},\end{cases}
$$

and

$$
F_{3}(x)= \begin{cases}\frac{x\left(1-\frac{v_{1}}{2 v_{3}}\right)+2 v_{1}-2 v_{3}}{v_{1}} & \text { for } x \leq 2 v_{3} \\ 1 & \text { for } x>2 v_{3}\end{cases}
$$

Case 3: If $v_{1}>v_{2}=v_{3}$, the unique Nash equilibrium is given by

$$
\begin{gathered}
F_{1}(x)= \begin{cases}0 & \text { for } x<\frac{2 v_{3}\left(v_{1}-v_{3}\right)}{2 v_{1}-v_{3}} \\
\frac{x}{v_{3}}-\frac{v_{1}-v_{3}+\frac{x}{2}}{v_{1}} & \text { for } \frac{2 v_{3}\left(v_{1}-v_{3}\right)}{2 v_{1}-v_{3}} \leq x \leq 2 v_{3} \\
1 & \text { for } x \geq 2 v_{3},\end{cases} \\
F_{2}(x)=F_{3}(x)= \begin{cases}\frac{x}{v_{3}} & \text { for } x<\frac{2 v_{3}\left(v_{1}-v_{3}\right)}{2 v_{1}-v_{3}} \\
\frac{v_{1}-v_{3}+\frac{x}{2}}{v_{1}} & \text { for } \frac{2 v_{3}\left(v_{1}-v_{3}\right)}{2 v_{1}-v_{3}} \leq x \leq 2 v_{3} \\
1 & \text { for } x \geq 2 v_{3} .\end{cases}
\end{gathered}
$$

Proof. Xiao (2016) analyzes all-pay auctions with multiple prizes and derives an algorithm for linear cost functions which can be used to determine the equilibrium under two assumptions, called A.1 and A.2. Assumption A.2 restricts the form of the prizes. In particular, if the difference between prizes 1 and 2 is the same as the difference between prizes 2 and 3 as we assumed here, the sequence of prizes is called a quadratic prize sequence. This is a sufficient condition to guarantee that Assumption A.2 holds.

Assumption A.1 says the marginal cost should be ordered, which is equivalent to our condition of constant ordering of valuations in Section 4 up to the fact that we allow for equal valuations. The construction of the unique equilibrium in Xiao (2016) extends for 
equal valuations and there may only be other additional equilibria when $v_{m+1}=v_{m+2}{ }^{8}$ Since this is not the case here, the equilibrium in the static model is unique and we can use Xiao's algorithm to obtain it. We provide the details in the appendix. This allows us to compare the total expected effort in the static and the two-stage contest.

Uniqueness follows from directly from Xiao (2016). Existence is straightforward to verify by checking that the payoffs of each player are constant on the respective support and lower for every other bid. We omit the straightforward calculations, but give an intuition for the different cases below.

In the first case $v_{1}=v_{2} \geq 2 v_{3}$, players 1 and 2 compete for the first prize, but are guaranteed the second prize since player 3 is inactive. The difference between first and second is $v_{1}$, the endpoint of the supports. Player 3 has such a low valuation that given the bids of players 1 and 2, any positive bid would yield a (weakly) negative payoff. Thus, he abstains from bidding.

In the second case, we still have two strong bidders, but the valuation of the weak bidder is relatively higher. Thus, player 3 randomizes on a common interval with players 1 and 2 , but also places probability mass at zero. Finally, in the case of two weak bidders, all three bidders randomize with positive probability on the same interval.

Proof of Proposition 7. Using Lemma 9, we can compare the static contest to the two-stage contests.

\section{Calculation of total expected effort:}

For two equally strong contestants, we have the effort distributions in the Nash equilibrium of the static contest and in the (symmetric and monotone) subgame-perfect equilibrium of the two-stage contest. This allows us to derive the total expected effort in each contest.

Consider the static game.

\footnotetext{
${ }^{8}$ This extends the reasoning of Baye, Kovenock, and de Vries (1996) for the case of one prize and at least three players where $v_{2}=v_{3}$.
} 
(i): If $v_{1}=v_{2} \geq 2 v_{3}$, contestant 3 remains inactive. Thus, we have

$$
T E=2 \int_{0}^{v_{1}} f_{1}(x) x d x=v_{1}
$$

(ii): If $v_{1}=v_{2}<2 v_{3}$, contestant 3 is active. For the total effort, we obtain

$$
T E=2 \int_{0}^{2 v_{3}} f_{1}(x) x d x+\int_{0}^{2 v_{3}} f_{3}(x) x d x=v_{3}+\frac{2 v_{3}^{2}}{v_{1}} .
$$

(iii): If $v_{1}>v_{2}=v_{3}$, contestant 3 is active. We have

$$
T E=\int_{\frac{2 v_{3}\left(v_{1}-v_{3}\right)}{2 v_{1}-v_{3}}}^{v_{3}} f_{1}(x) x d x+2 \int_{0}^{v_{3}} f_{3}(x) x d x=2 v_{3}+\frac{v_{1} v_{3}}{2 v_{1}-v_{3}} .
$$

Consider the two-stage contest.

(a): Let us first consider the case $v_{1}=v_{2}>v_{3}$. The total effort is given by

$$
\begin{array}{r}
T E=2 \int_{0}^{v_{3}} f_{1}(x) x d x+\int_{0}^{v_{3}} f_{3}(x) x d x+\frac{v_{3}^{2}}{2 v_{1}}+\frac{v_{3}}{2} \\
+\left(v_{1}-\left(\frac{v_{3}^{2}}{2 v_{1}}+\frac{v_{3}}{2}\right)\right)\left(F_{3}(0)+\int_{0}^{v_{3}} f_{3}(x)\left(1-F_{1}(x)\right)^{2} d x\right) .
\end{array}
$$

The first two terms in Eq. (3) are the expected efforts exerted in Round 1. By the construction in Baye, Kovenock, and de Vries (1996), the expected effort in Round 2 is $\frac{v_{3}}{2}+\frac{v_{3}^{2}}{2 v_{1}}$ if player 3 qualifies and $v_{1}>\frac{v_{3}}{2}+\frac{v_{3}^{2}}{2 v_{1}}$ if player 3 does not qualify. Thus, the expected efforts in Round 2 are $\frac{v_{3}}{2}+\frac{v_{3}^{2}}{2 v_{1}}$ plus $v_{1}-\frac{v_{3}}{2}+\frac{v_{3}^{2}}{2 v_{1}}$ whenever player 3 does not qualify which explains the remaining terms in Eq. (3). The equation can be simplified to

$$
T E=v_{1}+\frac{4 v_{3}}{3}+\frac{2 v_{3}^{2}}{3 v_{1}-v_{3}}-\frac{4 v_{1} v_{3}}{9 v_{1}-3 v_{3}}+\frac{2 v_{3}^{3}}{v_{1}\left(9 v_{1}-3 v_{3}\right)} .
$$

Putting everything on a common denominator, we obtain the equation in the proposition.

(b): Let us now consider the case $v_{1}>v_{2}=v_{3}$. The calculation of total effort is similar to the first case:

$$
T E=\int_{0}^{v_{3}} x f_{1}(x) d x+2 \int_{0}^{v_{3}} x f_{3}(x) d x+v_{3}+\left(\frac{v_{3}^{2}}{2 v_{1}}-\frac{v_{3}}{2}\right) \int_{0}^{v_{3}} f_{1}(x)\left(2 F_{3}(x)-\left(F_{3}(x)\right)^{2}\right) d x .
$$


This can be calculated as

$$
T E=\frac{v_{3}^{3}}{6\left(2 v_{1}-v_{3}\right)^{2}}+\frac{v_{3}^{2}}{3 v_{1}}+\frac{5 v_{3}}{2}
$$

\section{Comparison of total expected effort:}

Denote the difference between the total effort in the two-stage contest and the total effort in the corresponding static contest by $\Delta$. We have to show that $\Delta>0$ for all considered parameters. We distinguish three cases:

Case 1: Suppose $v_{1}=v_{2} \geq 2 v_{3}$. We obtain

$$
\Delta\left(v_{1}, v_{3}\right)=\frac{9 v_{1}^{3}+5 v_{1}^{2} v_{3}+2 v_{1} v_{3}^{2}+2 v_{3}^{3}}{3 v_{1}\left(3 v_{1}-v_{3}\right)}-v_{1}=\frac{2 v_{3}\left(4 v_{1}^{2}+v_{1} v_{3}+v_{3}^{2}\right)}{3 v_{1}\left(3 v_{1}-v_{3}\right)}>0 .
$$

Case 2: $v_{1}=v_{2}<2 v_{3}$. We obtain

$$
\Delta\left(v_{1}, v_{3}\right)=\frac{9 v_{1}^{3}+5 v_{1}^{2} v_{3}+2 v_{1} v_{3}^{2}+2 v_{3}^{3}}{3 v_{1}\left(3 v_{1}-v_{3}\right)}-v_{3}-\frac{2 v_{3}^{2}}{v_{1}}=\frac{9 v_{1}^{3}-4 v_{1}^{2} v_{3}-13 v_{1} v_{3}^{2}+8 v_{3}^{3}}{3 v_{1}\left(3 v_{1}-v_{3}\right)}
$$

Thus, it suffices to show that the numerator $\Delta_{n u m}=9 v_{1}^{3}-4 v_{1}^{2} v_{3}-13 v_{1} v_{3}^{2}+8 v_{3}^{3}$ is positive. At $v_{1}=v_{3}$, we have $\Delta_{\text {num }}=0$. Moreover, note that the derivative $\frac{d \Delta_{\text {num }}}{d v_{1}}=$ $27 v_{1}^{2}-8 v_{1} v_{3}-13 v_{3}^{2} \geq 6 v_{1}^{2}>0$ for all $v_{3}$ such that $v_{1} \geq v_{3}>0$. Thus, since $v_{1}>v_{3}$, we obtain $\Delta\left(v_{1}, v_{3}\right)>\Delta\left(v_{1}, v_{1}\right)=0$.

Case 3: Suppose $v_{1}>v_{2}=v_{3}$. We obtain

$$
\Delta\left(v_{1}, v_{3}\right)=\frac{v_{3}^{3}}{6\left(2 v_{1}-v_{3}\right)^{2}}+\frac{v_{3}^{2}}{3 v_{1}}+\frac{5 v_{3}}{2}-2 v_{3}-\frac{v_{1} v_{3}}{2 v_{1}-v_{3}}=\frac{v_{3}\left(v_{1}-v_{3}\right)^{2}}{3 v_{1}\left(2 v_{1}-v_{3}\right)^{2}}>0 .
$$


Proof of Proposition 8. Let us first consider the case $v_{1}=v_{2}>v_{3}$. Using the result in Clark and Riis (1998), the effort distributions (on the interval $\left.\left(0, v_{3}\right)\right)$ in Round 1 are given by $F_{1}(x)=F_{2}(x)=1-\left(1-\frac{x}{v_{3}}\right)^{0.5}$ and $F_{3}(x)=1-\frac{v_{3}}{v_{1}}\left(1-\frac{x}{v_{3}}\right)^{0.5}$.

Note that $F_{1}(x)$ and $F_{2}(x)$ are identical to the case with announcement. For $F_{3}(x)$, we have that $-\frac{2 v_{3}}{3 v_{1}-v_{3}}>-\frac{v_{3}}{v_{1}}$. Thus, player 3's distribution function with announcement is stochastically dominated by his distribution for the case without announcement. Clearly, this increases the probability that player 3 is eliminated in Round 1 and thereby increases the probability that a strong player wins in Round 2.

The total effort without announcement is given by $T E=\frac{4}{3} v_{3}+\frac{2}{3} \frac{v_{3}^{2}}{v_{1}}+v_{1}-\frac{2}{3} v_{3}+\frac{v_{3}^{2}}{3 v_{1}}+\frac{v_{3}^{3}}{3 v_{1}^{2}}$.

A comparison to the case with announcement shows the total effort is larger by announcing if $v_{3}<\frac{17^{0.5}-3}{2} v_{1}$.

It remains to consider the case $v_{1}=v_{2}>v_{3}$. Using the result in Clark and Riis (1998), the effort distributions in Round 1 are given by

$$
\begin{gathered}
F_{1}(x)= \begin{cases}0 & \text { for } x<v_{3}\left(1-\frac{v_{3}}{v_{1}}\right) \\
1-\left(\frac{v_{1}}{v_{3}}\right)^{0.5}\left(1-\frac{x}{v_{3}}\right)^{0.5} & \text { for } x \in\left[v_{3}\left(1-\frac{v_{3}}{v_{1}}\right), v_{3}\right] \\
1 & \text { for } x>v_{3},\end{cases} \\
F_{2}(x)=F_{3}(x)= \begin{cases}\frac{x}{v_{3}} & \text { for } x<v_{3}\left(1-\frac{v_{3}}{v_{1}}\right) \\
1-\left(\frac{v_{3}}{v_{1}}\right)^{0.5}\left(1-\frac{x}{v_{3}}\right)^{0.5} & \text { for } x \in\left[v_{3}\left(1-\frac{v_{3}}{v_{1}}\right), v_{3}\right] \\
1 & \text { for } x>v_{3} .\end{cases}
\end{gathered}
$$

The probability that player 1 qualifies is thus $1-\frac{v_{3}^{2}}{3 v_{1}^{2}}$, i.e., lower than in the case with announcement.

Expected total effort equals $T E=\frac{1}{6} v_{3}\left(\frac{v_{3}^{2}\left(3 v_{1}-v_{3}\right)}{v_{1}^{3}}+\frac{v_{3}}{v_{1}}+15\right)$. 
The calculation shows that TE is higher by announcing if

$$
v_{3}<\left(2-\frac{(9-\sqrt{78})^{\frac{1}{3}}}{3^{\frac{2}{3}}}-\frac{1}{3(9-\sqrt{78})^{\frac{1}{3}}}\right) v_{1} .
$$

\section{References}

Altmann, S., A. Falk, and M. Wibral (2012): "Promotions and Incentives: The Case of Multistage Tournaments," Journal of Labor Economics, 30, 149-174.

Arve, M., And O. Chiappinelli (2018): "The Role of Budget Constraints in Sequential Elimination Tournaments," Working Paper.

Baye, M. R., D. Kovenock, and C. G. De VRIEs (1993): "Rigging the Lobbying Process: An Application of the All-Pay Auction," American Economic Review, 83, 289294.

Baye, M. R., D. Kovenock, and C. G. De VRIEs (1996): "The all-pay auction with complete information," Economic Theory, 8, 291-305.

Clark, D. J., and C. RiIs (1998): "Competition over More than One Prize," American Economic Review, 88, 276-289.

Delfgaauw, J., R. Dur, A. Non, and W. Verbeke (2015): "The Effects of Prize Spread and Noise in Elimination Tournaments: A Natural Field Experiment," Journal of Labor Economics, 33, 521-569.

Fu, Q., And J. Lu (2012): "The optimal multi-stage contest," Economic Theory, 51, 251382.

Groh, C., B. Moldovanu, A. Sela, and U. Sunde (2012): "Optimal seedings in elimination tournaments," Economic Theory, 49, 59-80. 
Harris, C., AND J. ViCKers (1987): "Racing with Uncertainty," Review of Economic Studies, 54, 1-21.

Hillman, A. L., And J. G. Riley (1989): "Politically contestable rents and transfers," Economics and Politics, 1, 17-39.

Hillman, A. L., And D. Samet (1987): "Dissipation of contestable rents by small numbers of contenders," Public Choice, 54, 63-82.

Klose, B., And D. Kovenock (2015a): "The all-pay auction with identity-dependent externalities," Economic Theory, 59, 1-19.

Klose, B., And D. Kovenock (2015b): "Extremism drives out moderation," Social Choice and Welfare, 44, 861-887.

KonRAD, K. A. (2006): "Silent interests and all-pay auctions," International Journal of Industrial Organization, 24, 701-713.

Konrad, K. A., And D. Kovenock (2009): "Multi-battle contests," Games and Economic Behavior, 66, 256-274.

Moldovanu, B., And A. Sela (2006): "Contest architecture," Journal of Economic Theory, 126, 70-96.

Rosen, S. (1986): "Prizes and Incentives in Elimination Tournaments," American Economic Review, 76, 701-715.

Seel, C., And P. Strack (2016): "Continuous Time Contests with Private Information," Mathematics of Operations Research.

SiegEL, R. (2009): "All-pay contests," Econometrica, 77, 71-92.

XıAO, J. (2016): "Asymmetric all-pay contests with heterogeneous prizes," Journal of Economic Theory, 163, 178-221. 